\title{
Linking Continuum Mechanics and 3D Discrete Dislocation Simulations
}

\author{
M.C. Fivel \\ A.A. El-Azab
}

This paper was prepared for submittal to the 3rd European Mechanics of Materials Conference on Mechanics and Multiphysics Processes in Solids Oxford, United Kingdom

November 23-25, 1998

October 18, 1998

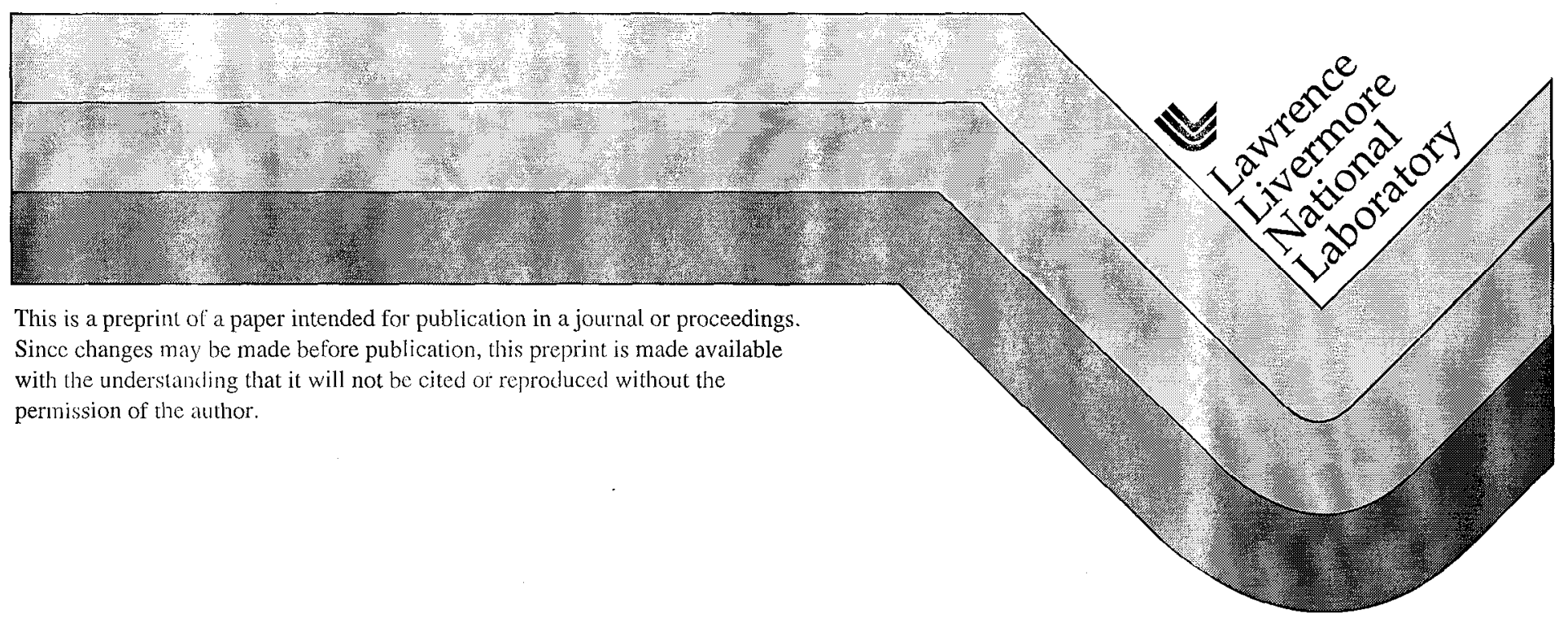




\section{DISCLAIMER}

This document was prepared as an account of work sponsored by an agency of the United States Government. Neither the United States Government nor the University of Califomia nor any of their employees, makes any warranty, express or implied, or assumes any legal liability or responsibility for the accuracy, completeness, or usefulness of any information, apparatus, product, or process disclosed, or represents that its use would not infringe privately owned rights. Reference herein to any specific commercial product, process, or service by trade name, trademark, manufacturer, or otherwise, does not necessarily constitute or imply its endorsement, recommendation, or favoring by the United States Government or the University of California. The views and opinions of authors expressed herein do not necessarily state or reflect those of the United States Govemment or the University of California, and shall not be used for advertising or product endorsement purposes. 


\title{
Linking continuum mechanics and 3D discrete dislocation simulations
}

\author{
M.C. Fivel ${ }^{\dagger *}$ and A.A. El-Azab ${ }^{\ddagger}$
}

†'GPM2 ENSPG, BP46, 101 Rue de la Physique, 38402 Saint Martin d'Hères Cedex, FRANCE.

present address : Lawrence Livermore National Lab., P.O. Box 808 L-415, Livermore CA 94550, USA.

FPacific Northwest National Laboratory, MSIN:K5-26, P.O. Box 999, Richland WA 99352, USA.

\begin{abstract}
A technique is developed for linking the methods of discrete dislocation dynamics simulation and finite element to treat elasto-plasticity problems. The overall formulation views the plastically deforming crystal as an elastic crystal with continuously changing dislocation microstructure which is tracked by the numerical dynamics simulation. The FEM code needed in this regard is based on linear elasticity only. This formulation presented here is focused on a continuous updating of the outer shape of the crystal, for possible regeneration of the FEM mesh, and adjustment of the surface geometry, in particular the surface normal. The method is expected to be potentially applicable to the nanoindentation experiments, where the zone around the indenter-crystal contact undergoes significant permanent deformation, the rigorous determination of which is very important to the calculation of the indentation print area and in turn, the surface hardness. Furthermore, the technique is expected to account for the plastic history of the surface displacement under the indenter. Other potential applications are mentioned in the text.
\end{abstract}

\section{INTRODUCTION}

In the past few years, 3D discrete dislocation dynamics models have been developed in order to study the plastic behavior of both fcc [1] and bcc [2] single crystals. These models bridge the gap between the now wcll-known behavior of a single dislocation [3-4] and the cooperative effects of a large population of dislocations on the macroscopic behavior of a single crystal. The length scale at which the simulation is performed is large enough for the theory of elasticity of dislocation fields to remain valid. The dislocation lines are discretized in pure edge and screw elements which have their own long-range stress field. The total, applied plus long-range, stress field determines the course of motion of these dislocation elements. Linking the mesoscopic simulation and continuum mechanics is achieved by calculation of continuous field variables from the simulation results. The way the simulation is conducted makes it straightforward to find the stress fields inside the crystal. In order to extract a smooth plastic strain field, and in turn, determine the shape of the deforming crystal, other mathematical conditions need to be satisfied. For example, the incremental plastic strain field is obtained by averaging the discrete incremental strain due to the motion of individual dislocation elements in a control volume. In order to obtain a spatially smooth plastic strain field within the simulated crystal space, it is required that the simulation volume have a size scale that is much larger than the size of the averaging volume. Unless this condition is satisficd, the smoothing of the plastic strain field will be strongly dependent on the choice of such control volume, and in turn, this strain field can not be integrated to yield a meaningful displacement field. If the purpose, however, is to determine the outer shape of the crystal, i.e., the deformed boundary, the present work shows that there may not be a need to obtain a smooth plastic strain field by performing averaging in the bulk of the crystal. Determination of the outer boundary shape of a finite crystal is necessary if part the crystal undergoes finite deformations, since the formulation 
(whether discrete or continuum) in this case requires continuous update of the boundary geometry, essentially, the changes in both magnitude and unit normal of differential surface elements. These requirements are critical to problem such as nano-indentation, where localized finite deformations take place around the contact zone.

In this paper, a technique is developed to establish the linkage between the discrete simulations and the continuum theory of deformation, focusing only of the geometry of the boundary of the deforming crystal. Essentially, an incremental formulation of the dislocation dynamics problem is achieved which is based on the following ideas:

1) the simulated crystal is an elastic crystal with moving dislocation and the dynamics of the dislocation system is governed by the both long-range and applied stress fields which are fully determined by the linear elasticity theory;

2) as dislocations move and cause plastic distortion, the boundary geometry is rigorously and incrementally updated;

3) updating the boundary geometry is not based on integrating a smooth distortion field in the bulk, but rather by considering only the permanent deformation at the surface. Such deformation is fully determined by the motion of the dislocation elements close to the boundary;

4) the stress ficld influcncing the dynamics of the dislocation system, specifically that part associated with boundary loading, is found based on continuously updated boundary geometry.

The next section exposes the basis of the 3D discrete dislocation modeling. The scheme of discretization of space and dislocation lines and the dynamics of the dislocation ensemble are described. In section 3, the boundary conditions imposed on the simulated volume are given. It is also shown how the simulation is conducted for both bulk and finite volume crystals undergoing infinitesimal plastic deformations. Section 4 includes a generalization of the principle adopted in the previous section and a proposition of a method to take into account the permanent deformation generated by the dislocation motion. Some remarks are then given on the utilization of this work within the context of multi-scale modeling of the plastic deformation.

\section{THE DISCRETE DISLOCATION MODELING}

\subsection{Discretization}

The discrete dislocation dynamics model described here is based on discretization of the simulated crystal space into a lattice with interior points between which the dislocation lines are presmissible. This rule minimizes the number of dislocation segments to deal with during the simulations. A natural way to discretize the space consists of defining a discrete network of points spread on a lattice similar to the crystal lattice but with a larger size. The size of this lattice is chosen so that two neighboring points are separated from a distance equal to the minimum distance below which two dislocation elements of opposite sense can not coexists without instantaneously annihilating each other. This distance can be inferred from experiments [5] and generally it is found to be on the order of $10 \times b$ where $b$ is the magnitude of the Burgers vector. Since this length scale represents the minimum possible distance between two interacting dislocation elements, the use of linear elasticity solutions for dislocation stress fields is justifiable since this separation is reasonably larger than the dislocation core size.

The dislocation lines are discretized into pure edge and screw segments, which are fitted to the lattice. This specificity simplifies the rules to use for the dislocation interactions since edge and screw segments are perpendicular to one another. For fcc simulations, it is needed to define eighteen discrete vectors (twelve $<112>$ edge and six $<110\rangle$ screw) for dislocation residing on twelve $\{111\}$ slip planes. For a bcc crystal, however, twelve $<112>$ edge and four $<111>$ screw vectors are needed to define the twelve $\{110\}$ slip planes. 


\subsection{Stress Computation}

The dislocation segments move in reaction to the effective shear stress resolved on their glide plane and computed at their center. This stress $\tau^{*}$ is written as

$$
\tau^{*}=[\sigma . \mathbf{b} \times \mathbf{I}] . \mathbf{b}+\tau_{\mathrm{lt}}-\tau_{\text {Peierls }},
$$

in which $\mathbf{b}$ and $\mathbf{l}$ are respectively the Burgers and the line vectors of the dislocation segment. $\tau_{\text {Peierls }}$ corresponds to the lattice friction generated by the Peierls forces, and $\tau_{\mathrm{lt}}$ represents the line tension effect. For fcc simulation this term is computed using the local curvature of the dislocation line

$$
\tau_{\mathrm{lt}}=\frac{\alpha \mathrm{Gb}}{\mathrm{R}}
$$

where $\mathrm{G}$ is the shear modulus, $\mathrm{R}$ is the local radius of curvature and $\alpha$ is a parameter defined experimentally. For bcc simulation, the line tension is computed using the gradient of the self energy as proposed by Devincre and Condat [6]. $\sigma$ is the local stress tensor computed at the center of each segment. Using the superposition principle, see Lubarda et al. [7], this tensor is decomposed into two parts

$$
\sigma=\hat{\sigma}+\tilde{\sigma}
$$

where the stress component $\hat{\sigma}$ is contributed by all other dislocation segments within the simulated box, as if they were in an infinite medium. It is computed using the de Wit formula [8], which is written in detailed in [9]. A method to expedite the computation is described in [10]. The field $\tilde{\sigma}$ is a stress component needed to enforce the boundary conditions. This component will be detailed in the following sections depending on the situation.

\subsection{Kinetics}

At a given time step, the dislocation configuration is frozen and the stress is computed on the middle points of each dislocation segment. The segments then move according to the following phonon drag viscous law

$$
\mathrm{v}=\tau^{*} \frac{\mathrm{b}}{\mathrm{B}}
$$

where $b$ is the magnitude of the Burgers vector, $\tau^{*}$ the effective resolved shear stress defined previously, and $\mathrm{B}$ the phonon drag coefficient.

All three-dimensional possible interactions among the dislocation segments such as the annihilation and junction formation, as well as cross-slip are taken into account. Annihilation events occur when two dislocation segments of the same Burgers vectors but with opposite signs occupy the same space on the discrete lattice. The common part is then removed and the two lines are re-ordained. Cross-slip events are possible for screw segments only and depending on a thermally-activated probabilistic law. If cross-slip is allowed, a screw segment is permitted to end its flight on a different slip system. Junction formation may occur when two dislocation segments that do not belong to the same glide plane meet in a 3D configuration. An energy criterion is used to determine if it is favorable to create a sessile junction and if so, both segments are blocked simulating a Lomer-Cottrell lock. Those junctions create the hard skeleton of the dislocation microstructure. They can be destroyed if the local resolved shear stress applied on at least one of those sessile segments is above a critical value found to be $12.5 \mathrm{MPa}$ for fcc materials by Devince and Kubin [11]. In the case of bcc materials, the stress needed to unzip a junction is not well known yet and the problem is currently under investigation. 


\section{THE BOUNDARY CONDITIONS}

\subsection{Overview}

Early simulations were performed without serious attention to the conditions on the boundary of the simulation volume. The purpose was to study the behavior of a bulk crystal and the simulation results were supposed to be statistically representative of the behavior of a much larger crystal. For simplicity, cubic simulation volumes were considered. The size of the cube was chosen to be reasonably larger than the wavelength of any expected dislocation pattern, e.g., cells. Inferring from experiments, simulation volume sizes of about 15 and 20 micrometers were considered for fcc and bcc crystals, respectively. In principle, only a minimum bound should be imposed on the size of the simulation box for the simulation results to be meaningful. The remotely applied stress field $\tilde{\sigma}$ has been considered uniform. The effects of truncating the crystal at the surface of the simulation volume were eliminated by extracting information from a sphere centered within the simulation space, and whose diameter is smaller than the cube size [12]. Recently, a selfconsistent formulation of both traction and flux boundary conditions of a simulated bulk crystal space was completed [13].

If the purpose is to directly compare dislocation microstructures to those observed experimentally, a finite crystal should be considered. In this case, the boundary of the simulation volume is the physical crystal surface. The traction boundary condition in this case must be determined by the experimental conditions. The absorption and/or emission of dislocations from the crystal surface gives rise to the boundary condition for the dislocation field. Therefore, the primary consideration in the case of bounded crystals is to structure the simulation models to deal with complex boundary conditions. The main objective of the present work is indeed to outline the method proposed to deal with this kind of problem.

\subsection{Finite Crystal Loading}

Consider a simulation volume $\Omega$ which has an arbitrary shape as pictured in figure 1 . Assume that this volume contains dislocations and is submitted to forces $F^{\text {app }}$ on its boundary $d \Omega$. The superposition principle is used to determine the stress field $\sigma$ to be applied on the dislocation segments. As explained in the previous section (see also reference [12]), this stress field is decomposed into two contributions $\hat{\sigma}$ and $\tilde{\sigma}$. The first contribution $\hat{\sigma}$ is the stress field generated by the dislocations as if they were in an infinite medium. However, the residual traction $F^{\text {disl }}$ left by dislocations on the boundaries of the simulated volume needs to be canceled for the dislocation stress field alone to satisfy traction-free boundary conditions. It is therefore necessary to solve a problem where forces $F^{\mathrm{app}}-\mathrm{F}^{\mathrm{disl}}$ are applied on the boundary $\mathrm{d} \Omega$ of volume $\Omega$. The stress field $\tilde{\sigma}$ solution of this problem can be computed numerically using the boundary element or the finite element method. Here, a finite element algorithm is implemented and coupled with the discrete simulations. 


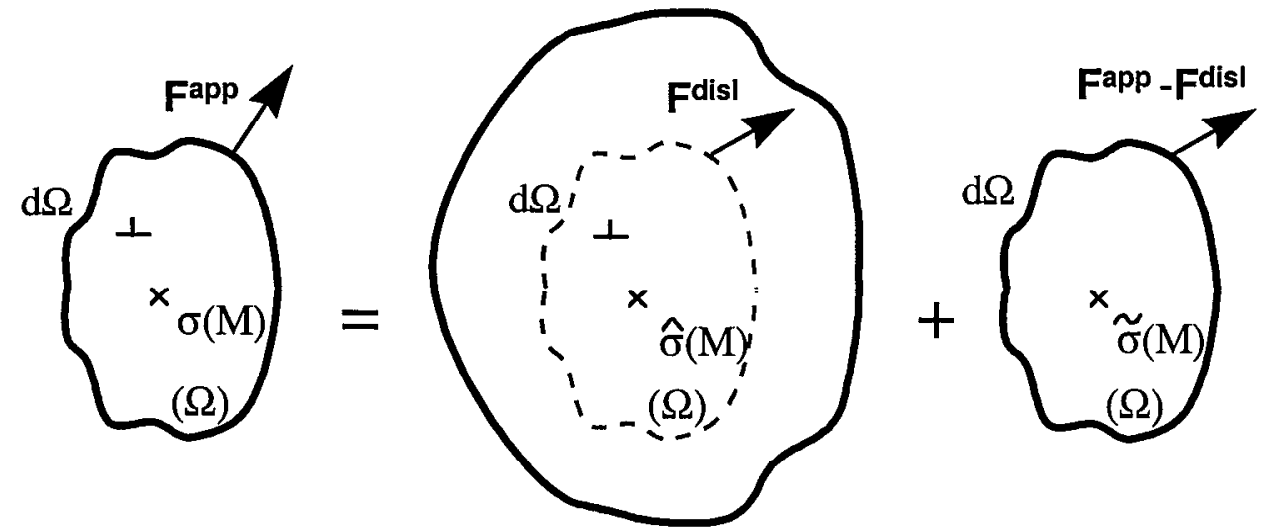

Figure 1: Decomposition of the boundary problem into two sub-problems. The stress field applied to the dislocation segments is given by superposition: $\sigma=\hat{\sigma}+\tilde{\sigma}$.

An application of this method has already been performed on the simulation of the nano-indentation test using the commercial FEM code CASTEM2000 [14]. The dislocation microstructure generated under the application of a spherical indenter on a pure [001] single crystal of copper has been simulated and dircctly compared to the experimental one. The simulated box consisted of a 2 microns large cube for which a part of the top surface was submitted to the parabolic Hertz loading corresponding to a spherical contact whereas the remaining top surface was imposed to be traction free. A set of nucleation rules defining the shape, size and location of the dislocation loops generated during the indentation process was determined using experimental data. The goal being to compare the simulated dislocation microstructure to the actual experimental one, the experimental conditions relevant to the thin foil extraction and the observation techniques have also been numerically reproduccd. Once performed, the comparison showed a good accuracy between both the shape and size of the plastic volume. These initial results indicate that the analysis of indentation induced plasticity on the basis of dislocation mechanics is a promising approach. However, it should be noted that the developed model has its own limitations in studying the nano-indentation test.

\subsection{Limitations of the Method}

The method described above, devised to account for the boundary conditions in the discrete dislocation code, is limited by being restricted to elastic deformation only. Indeed, in the case of the simulation of the nano-indentation the numerical code was not able to give a value of the hardness -as measured experimentally (force divided by the area of the indent print)- since the indent print could not be simulated. In fact, all the finite element solutions are always defined on the same geometry of the volume i.e. the boundary $\mathrm{d} \Omega$ does not change with loading, due to the fact that dislocations were envisioned to move in a linearly elastic solid with no account for permanent deformations. When these defects approach the boundary of the simulated volume, they were systematically eliminated from the simulation and no history of the deformation is kept. In other words, since the plastic deformation corresponding to the steps created by the outgoing dislocations is not simulated, the boundary of the volume remains the same from the beginning to the end of the simulation. In the case of the nano-indentation simulation, this means that the size of the indent print at a given indentation depth is just linked to the dislocations which are still inside the volume and don't take into account all the dislocations which have disappeared on the boundary surfaces.

Based on the previous argument, accounting for the plastic deformation of the finite crystal in the hybrid code would allow access to information that is essential to comprehension of the nano-indentation test. Examples are the indent size and shape, the hardness and the unloading curve. More generally, such an implementation would be a paradigm for linking between the mesoscopic and macroscopic scales. 


\section{CONSIDERATION OF PLASTIC DEFORMATION}

\subsection{Problematic and intuitive solution}

A segment of length $\mathrm{L}$, moving on a distance $\mathrm{dx}$ at a velocity $\mathrm{v}$, over the time step $\delta \mathrm{t}$ sweeps an area $\mathrm{dA}=\mathrm{L} . \mathrm{dx}$ which gives raise to the incremental shear strain $\Delta \gamma$ given by the Orowan law

$$
\Delta \gamma=\rho_{\mathrm{m}} \mathrm{bv} \delta \mathrm{t}=\frac{\mathrm{L}}{\mathrm{V}} \mathrm{bdx}=\mathrm{b} \frac{\mathrm{dA}}{\mathrm{V}},
$$

in which $\mathrm{V}$ is the volume within which this plastic deformation occurs. The plastic distortion increment is then deduced from the shear strain on all the glide systems, using the relation

$$
\Delta \beta_{\mathrm{ij}}^{\mathrm{p}}=\sum_{\text {segments }} \mathrm{b}_{\mathrm{i}} \mathrm{n}_{\mathrm{j}} \Delta \gamma \text {. }
$$

The plastic strain and the rotation increments are deduced from equation (6) by taking the symmetric and skew parts of $\Delta \beta^{p}$, respectively. Equation (6) applies to produce an averaged plastic distortion at a continuum point in a large crystal. Within the simulation framework this continuum point is just the simulation volume, if the purpose is to simulate a bulk crystal. If a bounded crystal is to be simulated, caution must be exercised when applying equation (6), i.e., in generating a smooth plastic distortion field whose local value is found using that equation. The reason is that, the volume $\mathrm{V}$ must be much smaller, by at least an order of magnitude, than the size of the simulated crystal. However, if the simulated crystal has at least one dimension on the order of the size of volume necessary to apply equation (6), then constructing a smooth plastic distortion field is meaningless. In this case, the simulated crystal can not be dealt with as a continuum elastoplastic solid. Specifically, the boundary value problem can not be solved by, say, an FEM method, given the fact that the plastic strain field is calculated from the simulations. An attempt has been made by Lemarchand et al. [15] to construct smooth plastic distortion fields from the evolution of the simulated dislocation ensemble in a bulk crystal. Here we approach this problem from a different angle, and the formulation presented here shows that it may be possible to avoid constructing a smooth plastic distortion field using the simulation information.

The crystal is viewed here to be elastic at all times, and the motion of dislocations can be viewed in two ways; introduction of plastic strain increments which give rise to increments in the elastic fields, or a transition from one elastic state to another. In either view, the plastic distortion associated with motion of dislocations is manifested in the change of the elastic state of the crystal. Assume that we know the state of stress and elastic distortion at time $t$, and following minor rearrangement of the dislocation structure, it is required to determine the new stress and distortion fields in the crystal at $t+\delta t$. One approach is to use the incremental plastic distortions (eigenstrain) in a formal elasticity solution and obtain a stress component, which represents the difference between the stress states at $t$ and $t+\delta t$. Associated with this stress increment is an elastic distortion increment, which again is the difference between the elastic distortion states at $t$ and $t+\delta t$. In this manner, the current elastic state is dependent on the history of plastic deformation. However, as explained previously, a quasi-continuous plastic strain field must be constructed.

Another approach is to determine the current elastic distortion and stress states directly from the current dislocation content of the crystal, by considering the dislocation structure to be a state variable. This is consistent with the notion that the history of a plastically deforming crystal is a sequence of elastic crystals, and the motion of dislocations yields the transition from one crystal to the other within this sequence. In this way, the plastic distortion $\Delta \beta^{p}$, as defined in equation (6), need not be taken into account in generating the stress field of in the crystal, as long as the dislocation content is known. This notion fits quite well within the structure of the method of dislocation dynamic simulation. However, the question to be asked is as follows: can one recover a meaningful displacement field in the crystal, and can this field exist everywhere in the dislocated crystal? This question is answered in the rest of this presentation. 


\subsection{A Proposed Boundary Solution}

The theory of continuous distribution of dislocations is recalled here; see for example Mura [16]. In this classic formulation, total crystal distortion, $\beta^{\mathbf{T}}$ is decomposed into three components (see Figure 2),

$$
\beta_{\mathrm{ij}}^{\mathrm{T}}=\beta_{\mathrm{ij}}^{\circ}+\beta_{\mathrm{ij}}^{\mathrm{d}}+\beta_{\mathrm{ij}}^{\mathrm{p}}
$$

in which $\beta^{\circ}$ is an elastic distortion associated with the boundary load, $\beta^{\mathrm{d}}$ is the elastic distortion due to dislocations, and $\beta^{\mathrm{p}}$ is the plastic distortion. For the case of discrete simulations, the increments of the later are given by dirac-delta distributions localized on the dislocation lines.

The compatibility condition for total distortion is written in terms of the dislocation density tensor $\alpha$ and the plastic distortion $\beta^{\mathrm{p}}$ as follows:

$$
\mathrm{e}_{\mathrm{ikl}}\left(\beta_{\mathrm{j}, \mathrm{k}}^{\mathrm{d}}+\beta_{\mathrm{ij}, \mathrm{k}}^{\mathrm{p}}\right)=\alpha_{\mathrm{ij}}+\mathrm{e}_{\mathrm{ikk}} \beta_{\mathrm{ij}, \mathrm{k}}^{\mathrm{p}}=0,
$$

where $\beta^{\circ}$ is curl-free, and $e_{i k l}$ is the permutation tensor. Integrating (8) over the entire volume and using Gauss theorem,

$$
\int_{\Omega}\left(\alpha_{\mathrm{ij}}+\mathrm{e}_{\mathrm{ikl}} \beta_{\mathrm{j}, \mathrm{k}}^{\mathrm{p}}\right) \cdot \mathrm{dV}=\int_{\Omega} \alpha_{\mathrm{ij}} \cdot \mathrm{dV}+\int_{\mathrm{d} \Omega} \alpha_{\mathrm{ij}}^{\mathrm{s}} \cdot \mathrm{dS}
$$

where $\alpha_{i j}^{s}=e_{i k l} n_{k} \beta_{\mathrm{lj}}^{p}$ is the surface dislocation density tensor. Equations (8) and (9) are valid in a rate form.

The total distortion field is derivable from a continuous displacement field (so is $\beta^{\circ}$ since it is associated with boundary traction $\mathrm{F}^{\mathrm{app}}$ ). This displacement field can be constructed in the following manner,

$$
\int_{A B} \mathrm{~d} \mathbf{u}=\mathbf{u}(B)-\mathbf{u}(\mathrm{A})=\int_{A B} \mathrm{~d} \mathbf{x} \cdot \boldsymbol{\beta}^{\mathrm{T}}=\int_{A B} \mathrm{~d} \mathbf{x} \cdot\left(\boldsymbol{\beta}^{\circ}+\boldsymbol{\beta}^{\mathrm{d}}+\boldsymbol{\beta}^{\mathrm{P}}\right) .
$$

The integral is independent of the path followed from point A to B in the crystal. The displacement field constructed in this way is unique if the integral in (10) taken over a closed line identically vanishes. Also, the points A and B can be any two points on the surface of the crystal.
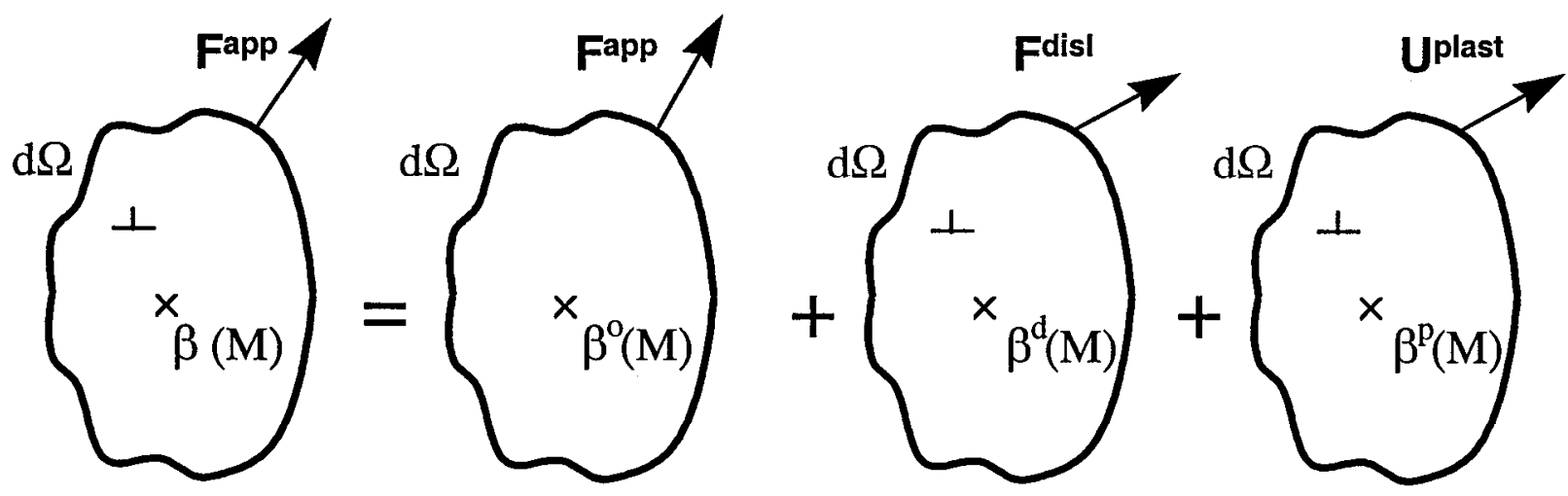

Figure 2: The total distortion is the summation of the elastic distortion field $\beta^{0}$ generated by the applied load, the distortion field $\beta^{\mathbf{d}}$ gencrated by the dislocations (which depends on their position) and the plastic distortion field $\beta^{\mathbf{p}}$.

\subsubsection{A crystal with bulk dislocations}

Assume that the plastic distortion field is vanishing on the crystal surface. From equation (10), the difference between the displacements of two points on the surface is given by

$$
\int_{A B(d \Omega)} d \mathbf{u}=\mathbf{u}(B)-\mathbf{u}(A)=\int_{A B(d \Omega)} d \mathbf{x} \cdot\left(\boldsymbol{\beta}^{\circ}+\beta^{d}\right)
$$


which implies that the elastic distortion field due to the presence of dislocations can be integrated on the surface to yield the shape of the crystal. In other words, given only the dislocation content of the crystal it is guaranteed that the outer shape of the crystal is uniquely determinable. Within the framework of simulations, superposition of the elastic distortion fields calculated using conventional elasticity solution can be used for this purpose. Equation (11) also implies that

$$
\oint_{C(d \Omega)} \mathbf{d x} \cdot \beta^{\mathbf{d}}=\mathbf{0}
$$

for any closed line $C$ on the surface of the crystal. It is important to mention here that the component $\beta^{\mathrm{d}}$ is not generally integrable in the bulk of the crystal. Its integration along a closed line yields the resultant Burgers vector of all dislocations passing through any area bounded by this line. See Figure 3 for an illustration.

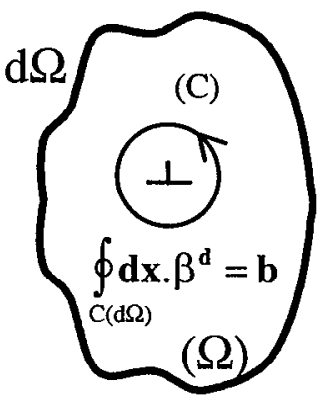

(a)

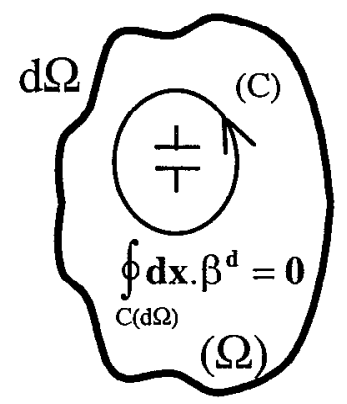

(b)

Figure 3: Integration of $\beta^{\mathbf{d}}$ doesn't give a unique displacement field in (a) whereas it gives a meaningful field when the path is chosen around a region containing a zero net Burgers vector.

The general conclusion to be made here is as follows:

"if a closed surface can be found in a plastically deformed crystal such that the plastic distortion is vanishing everywhere on this surface, it is possible to uniquely determine the shape of that surface at all times, knowing only the elastic distortion field created by dislocations at all points on that surface. Also, the resultant Burgers vector of all dislocations threading an arbitrary open area on this surface is equal to zero". The second part of this conclusion is satisfied if dislocations do not penetrate this surface at all.

\subsubsection{Taking into account the plasticity at the surfaces}

As implied by equation (9), a free surface can store dislocations, the tensor density of which is given by $\alpha_{\mathrm{ij}}^{\mathrm{s}}=\mathrm{e}_{\mathrm{ikl}} \mathrm{n}_{\mathrm{k}} \beta_{\mathrm{lj}}^{\mathrm{p}}$. The rate form of equation (9) is written as

$$
\int_{\Omega}\left(\dot{\alpha}_{\mathrm{ij}}+\mathrm{e}_{\mathrm{ikk}} \dot{\beta}_{\mathrm{lij}, \mathrm{k}}^{\mathrm{p}}\right) \cdot \mathrm{dV}=\int_{\Omega} \dot{\alpha}_{\mathrm{ij}} \cdot \mathrm{dV}+\int_{\mathrm{d} \Omega} \dot{\alpha}_{\mathrm{ij}}^{\mathrm{s}} \cdot \mathrm{dS}=0
$$

which can be thought of as a conservation law of the total Burgers vector in a deforming crystal. Equation (13) means that during plastic deformation the volume and bulk of the crystal exchange dislocations, however, the total sum of their Burgers vector is identically invariant. This can be easily visualized by imagining and edge dislocation (in a two-dimensional crystal) being sucked into the surface. While the bulk becomes dislocation-free, a stcp is created on the crystal surface, which carries the same Burgers vector. However, this is not the only mechanism of change of both populations. There is a difference though between bulk and surface dislocations. The former are associated with a stress field, and latter are not, i.e., surface dislocations are completely impotent. One more difference is that, the line sense of a surface dislocation always lies in the crystal surface, i.e., orthogonal to the surface normal. 
When dislocations reach the boundary $d \Omega$, their motion generally create steps on the surface. Those steps are essentially a result of non-vanishing plastic distortion at the crystal surface. Indeed they are closely connected to a surface displacement gradients, see the previous definition of the surface dislocation tensor. In order to find the shape of the distorted crystal in this case, those steps must be taken into account.

Let us denote the displacement gradient associated with surface steps by $\beta^{s}$. In this case, equation (10) can be rewritten in the form

$$
\int_{A B} d \mathbf{u}=\mathbf{u}(B)-\mathbf{u}(A)=\int_{A B} d \mathbf{x} \cdot \boldsymbol{\beta}^{T}=\int_{A B} d \mathbf{x} \cdot\left(\boldsymbol{\beta}^{\circ}+\boldsymbol{\beta}^{d}+\boldsymbol{\beta}^{s}\right) .
$$

The integration can only be performed on the outer surface of the crystal. Note that $\beta^{\mathrm{d}}$ cannot be integrated alone on this surface but the sum $\left(\beta^{d}+\beta^{s}\right)$ can, and its integration over a line from $A$ to $B$ yields the differential displacement between these two points resulting from the dislocation field alone.

Implementation of these concepts within the framework of dislocation dynamics simulations can be done incrementally. Over a certain period of time, the change in the surface dislocation density is related to the incremental change in stress-free surface displacement gradient (due to surface steps) by $\Delta \alpha_{i j}^{s}=e_{i l k} n_{1} \Delta \beta_{j k}^{s}=e_{i l k} n_{1} \frac{\partial \Delta u_{j}^{s}}{\partial x_{k}}$. The problem will be solved when $\Delta \alpha^{s}$ is determined everywhere on the boundary $d \Omega$. For each dislocation, the increment of the plastic distortion is considered to be localized on the dislocation line itself with the following expression

$$
\Delta \boldsymbol{\beta}^{\mathrm{s}}=(\mathrm{vdt} \times \mathrm{l}) \otimes \mathrm{b} \quad \text { or, } \quad \dot{\beta}_{\mathrm{ij}}^{\mathrm{s}}=\mathrm{e}_{\mathrm{ikl}} \mathrm{v}_{\mathrm{k}} \mathrm{l}_{\mathrm{l}} \mathrm{b}_{\mathrm{j}}
$$

This term is completely determined by the geometry of the interaction of the outgoing dislocation at the boundary. Figure 4 shows a schematic of one of these events.

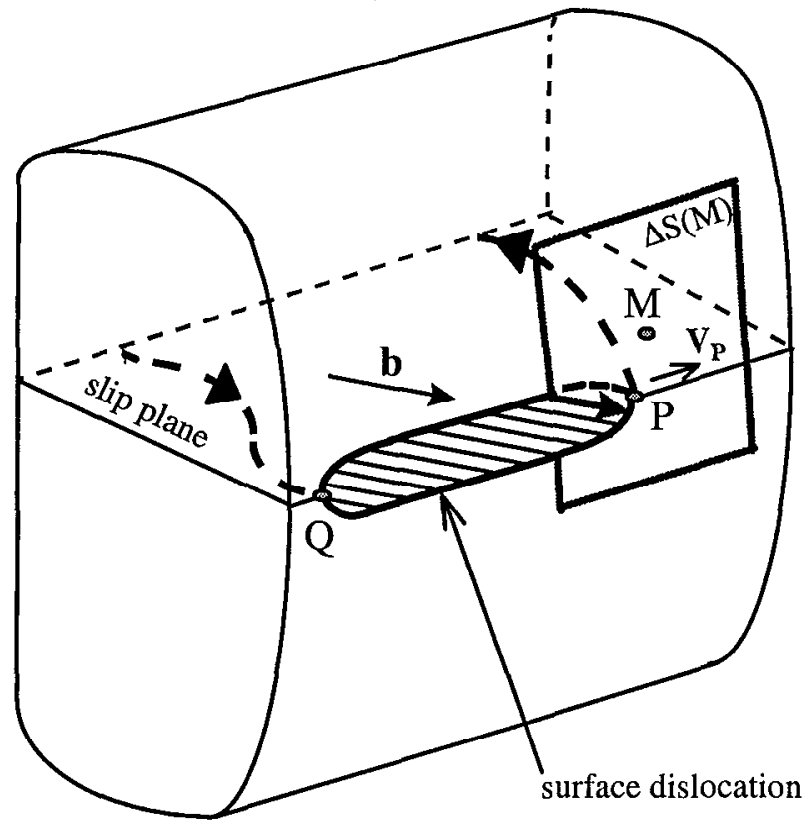

Figure 4: Scheme of the intersection of a dislocation line and a boundary surface.

A dislocation line has reached the surface and generated a slip trace there. As shown in that figure, the velocity of the point $P$, where the dislocation line intersects the crystal surface, determines the rate of creation of the slip trace at the surface. The velocity of point $P, v_{P}$ can be interpreted as the velocity at which points on both sides of the slip plane are shifted by an amount $\mathbf{b}$ relative to each other. The trace length created on a time increment $\delta \mathrm{t}$ is then $\Delta \mathbf{L}=\mathbf{v}_{\mathrm{p}} \delta \mathrm{t}$. This length indeed represents a surface dislocation. One can then define 
a similar length for each slip system. The tensor product of this length and the Burgers vector gives rise to a contribution to the surface dislocation density tensor. For a surface element $\Delta S$ in the neighborhood of some point $\mathrm{M}$ on the crystal boundary, the oriented dislocation length per unit area, which is accumulated over time $\delta \mathrm{t}$ is given by

$$
\Delta \mathrm{l}=\sum_{\text {all intersections }} \frac{\Delta \mathbf{L}}{\Delta \mathrm{S}}
$$

so that the contribution from all the systems to the increment of $\Delta \alpha^{\mathrm{s}}$ can be written as

$$
\Delta \boldsymbol{a}^{\mathrm{s}}=\sum_{\text {all slip systems }} \Delta \mathbf{l}^{(\mathrm{i})} \otimes \mathbf{b}^{(\mathrm{i})} \quad \text { or } \quad \Delta \alpha_{\mathrm{kl}}^{\mathrm{s}}=\sum_{\text {system }}^{\mathrm{N}} \Delta \mathrm{l}_{\mathrm{k}}^{(\mathrm{i})} \mathrm{b}_{1}^{(\mathrm{i})} .
$$

The incremental surface distortion due to creation of surface dislocations in the neighborhood of a point $\mathrm{M}$ on the crystal surface is related to the incremental change in the surface dislocation density tensor by

$$
\Delta \alpha_{i j}^{s}=e_{i l k} n_{1} \Delta \beta_{k j}^{s}=e_{i l k} n_{1} \frac{\partial \Delta u_{j}^{s}}{\partial x_{k}}
$$

which is a system of 9 equations for 9 unknowns gradient components. Once solved for $\Delta \beta_{\mathrm{kj}}^{\mathrm{s}}$, the latter can be substituted into equation (14) which can then be integrated to yield the incremental displacement field on the crystal surface.

The overall framework can be implemented as follows:

1) divide the surface into small elements;

2) track the creation of surface dislocations over incremental time periods;

3) calculate the incremental plastic distortion from the incremental surface dislocation density;

4) update the crystal boundary shape by integrating the incremental form of equation (14);

5) update the FEM mesh and apply the next boundary load increment;

6) calculate the long-range stress field and the stress field due to boundary load;

7) update the dislocation microstructure using dynamic simulations;

8) repeat steps 1 ) through 7).

\section{CONCLUDING REMARKS}

The notion that a plastically deformed crystal is an elastic crystal with moving defects is the basis of the present formulation. This notion is in fact naturally built in the method of dislocation dynamics simulation. The most important consequence of dealing with the crystal from this point of view is that the distortion field in the crystal can be determined by the spatial distribution of the dislocation elements, exactly in the same manner the elastic stress field is found. In other words, the change in plastic distortion of the crystal is manifested in the form of elastic distortion changes. However, generally speaking, neither the plastic nor the elastic distortions is individually integrable to yield a meaningful displacement field. However, only under certain conditions the integrability criterion is satisfied and it is possible to generate a meaningful displacement field only at the crystal surface. A displacement field is constructed on the crystal surface which has two components; an elastic component fully determined by the bulk dislocation structure, and a plastic component which is determined by surface dislocations. The later dislocation category simply represents the net creation of surface steps on the crystal as dislocations touching the surface glide on their slip planes. As shown in the last section, the method developed here does not rely on constructing or integrating a smooth plastic strain field.

When implemented in discrete dislocation codes, the method described here will allow the creation of a full hybrid code linking the actual deformation state of any single crystal to the behavior of the dislocation 
moving inside the simulated box. Direct results can be performed on the simulation of the nano-indentation test for which both the hardness and the unloading curve can be investigated by the new numerical code. Another direct application of the code can be done on the analysis of problems such as cavities growth.

The hybrid code can also be viewed as a usual finite element code for which the plastic strain evolution law has been removed and replaced by the dislocation dynamics simulation.

\section{Acknowledgments}

This work was performed under the auspices of the U.S. department of Energy by Lawrence Livermore National Laboratory under contract number W-7405-ENG-48.

\section{References}

[1] Kubin L.P., Canova G.R., Condat M., Devincre B., Pontikis V. and Bréchet Y., Solid State Phenom. 2324 (1992) 455-472.

[2] Tang M., Kubin L.P. and Canova G.R., Acta Mater. 46(9) (1998) 3221-3235.

[3] Friedel J., Dislocations (Pergamon Press, 1964).

[4] Hirth J.P. and Lothe J., Theory of dislocations ( $2^{\text {nd }}$ Ed. John Wiley \& Sons, 1982).

[5] Essmann U. and Mughrabi H., Phil. Mag. A40 (1979) 731-756.

[6] Devincre B. and Condat M., Acta Metall. Mater. 40 (1994) 2628-2637.

[7] Lubarda V.A., Blume J.A. and Needleman A., Acta Metall. Mater. 41 (1993) 625-642.

[8] DeWit R., Phys. State Solid. 20 (1967) 575-580.

[9] Devincre B., Solid State Com. 93 (1995) 875-878.

[10] Verdier M., Fivel M. and Groma I., Modelling Simul. Mater. Sci. Eng. (in press).

[11] Devincre B. and Kubin L.P., Modelling Simul. Mater. Sci. Eng. 2 (1994) 559-570.

[12] Fivel M.C. and Canova G.R., J. Phys. IV 8(Pr4) (1998) 249-258.

[13] El-Azab A., Eng. Report N UCLA/MATMOD-97-02, University of California, Los Angeles (1997).

[14] Fivel M.C., Robertson C.F., Canova G.R. and Boulanger L., Acta Mater. (in press).

[15] Lemarchand C., Chaboche J.-L., Devincre B. and Kubin L.P., submitted to this issue of J. Phys. IV.

[16] Mura T., Micromechanics of Defects in Dolids ( $2^{\text {nd }}$ Ed. Kluwer Academic Publisher Group, 1987). 
